\title{
Does Donor Age of Nonirradiated Achilles Tendon Allograft Influence Mid-Term Results of Revision ACL Reconstruction?
}

\author{
Stefano Zaffagnini ${ }^{1,2}$ Tommaso Roberti di Sarsina ${ }^{1,2}$ Tommaso Bonanzinga ${ }^{1,2}$ Marco Nitri ${ }^{1,2}$ \\ Luca Macchiarola $^{1,2}$ Federico Stefanelli ${ }^{1,2}$ Gianandrea Lucidi ${ }^{2}$ Alberto Grassi ${ }^{1,2}$ \\ ${ }^{1}$ Il Clinica Ortopedica e Traumatologica, Istituto Ortopedico Rizzoli, \\ Bologna, Italy \\ ${ }^{2}$ Laboratorio di Biomeccanica ed Innovazione Tecnologica, Istituto \\ Ortopedico Rizzoli, Bologna, Italy \\ Address for correspondence Tommaso Roberti di Sarsina, MD, \\ Istituto Ortopedico Rizzoli, Laboratorio di Biomeccanica ed \\ Innovazione Tecnologica, Via di Barbiano 1/10, 40136, Bologna, Italy \\ (e-mail: tommaso.robertidisarsina@hotmail.com).
}

Joints 2018;6:10-15.

\begin{abstract}
Purpose The purpose of the present study was to investigate if the donor age of nonirradiated Achilles tendon allograft could influence the clinical results of revision anterior cruciate ligament $(\mathrm{ACL})$ reconstruction.

Methods All patients that underwent ACL revision between 2004 and 2008 with at least 4 years of follow-up were included. For all the patients that met the inclusion criteria, the age of the graft donor was obtained from the tissue bank. Lysholm score was administered to patients that met inclusion criteria. In addition, patients were divided in two groups based on the donor age ( $<45$ years vs. $\geq 45$ years), and the baseline characteristics and outcomes were compared.

Results Fifty-two patients were evaluated at a mean $4.8 \pm 0.8$ years follow-up with Lysholm score. The Lysholm significantly improved from $62.3 \pm 6.6$ at preoperative status to $84.4 \pm 12.3$ at final follow-up. The mean donor age was $48.7 \pm 8.4$ years; a significant difference in Lysholm score was noted between patients that received an allograft with a

\section{Keywords}

- allograft

- Achilles tendon

- knee

- revision

- anterior cruciate ligament donor age $<45$ years ( 14 patients; $27 \%$ ) and those receiving an allograft with a donor age $\geq 45$ years $(38 ; 73 \%)(89.5 \pm 3.2$ vs. $80.1 \pm 11.1$, respectively; $p=0.0469)$. The multiple regression model showed the donor age, the final follow-up, and the preoperative Lysholm score as significant predictors of postoperative Lysholm score $(p<0.0002)$.

Conclusion Donor age of nonirradiated Achilles tendon allograft influenced the mid-term results of revision $\mathrm{ACL}$ reconstruction, thus advising the use of grafts from young donors. Level of Evidence Level III, retrospective comparative study.
\end{abstract}

\section{Introduction}

The use of allograft tissue for anterior cruciate ligament (ACL) reconstruction has been popularized in the last two decades. Its increased availability, in addition to the numerous advantages like extreme versatility, possibility of bone grafting, and no donor site morbidity, made the allograft tissue an appealing alternative to autograft for primary ACL recon-

received

August 22, 2017

accepted after revision

January 2, 2018

published online

February 12, 2018 struction and especially for ACL revision. The American Association of Tissue Banks reports that the demand for musculoskeletal grafts has grown from nearly 700,000 grafts in 2001 to approximately 1.5 million distributed in 2007, while the American Academy of Orthopaedic Surgeons has estimated that approximately 60,000 allografts were used in knee reconstruction procedures alone in $2005 .^{1,2}$ Furthermore, the multicentric ACL revision study reported that

Copyright $\odot 2018$ Georg Thieme Verlag License terms KG Stuttgart · New York

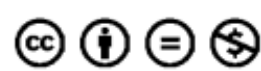


allografts were used in $27 \%$ of primary ACL reconstruction and in $54 \%$ of ACL revision. ${ }^{3}$ Despite good results have been reported in both these circumstances, ${ }^{4-8}$ the use of allograft is believed to produce inferior outcomes compared with autograft ${ }^{9}$ probably due to the slower incorporation process, ${ }^{10-12}$ the risk of disease transmission and immunological reaction, ${ }^{13,14}$ and the compromised biomechanical proprieties related to sterilization process. ${ }^{15}$

However, there are numerous variables like graft type, mechanical properties, surgical technique, sterilization method, follow-up, and patients characteristics, which could influence the results after ACL reconstruction with allograft. ${ }^{16-19}$ Regarding the age of graft donor, some advocate choosing tissue from younger donors on the premise that younger donors will have stronger tissue and, therefore, improved clinical results; however, controversies remain on this issue, due to the few discordant evidences from both in vitro and in vivo studies. ${ }^{20-25}$

The purpose of the present study was to investigate risk factors that could influences mid-term clinical results of revision ACL using nonirradiated Achilles tendon allograft, particularly the allograft donor age. The hypothesis was that higher age of graft donor was associated with worse subjective clinical results, after double-bundle ACL revision with nonirradiated Achilles tendon allograft.

\section{Methods}

All patients that underwent revision ACL between 2004 and 2008 were included in the study. Inclusion criteria were isolated or combined revision ACL with Achilles tendon allograft, age $>18$ years, no knee malalignment or knee malalignment corrected at time of indexed surgery or within 6 months from the indexed surgery. Exclusion criteria were follow-up $<4$ years and contralateral unstable or operated knee. For all the patients that met the inclusion criteria, the age of the graft donor was obtained from the tissue bank.

The patients that presented all the required characteristics to be involved in the study were contacted by phone by one investigator, which administered the Lysholm score. The Lysholm score is a patient-reported clinical score composed by eight multiple-answer questions that evaluate the limping due to knee pathologies, swelling, pain, instability, locking and giving-way sensation. ${ }^{26}$ The score ranges from 0 to 100 , where 0 represents complete disability and 100 represents optimal knee function. The Lysholm score was administered both at final follow-up and at preoperative status based on patient recall. All the data regarding age at primary reconstruction, age at revision, age at final follow-up, time from primary reconstruction to revision surgery, meniscal status, final follow-up, more than one prior ACL reconstruction, and combined procedures (excluding meniscectomy) were collected as well from the medical charts.

Patients were divided in two groups based on the donor age. Since Swank et $\mathrm{al}^{25}$ reported a decrease in ultimate tensile strength of allograft after the age ranging from 40 to 50 years, the mean value of this range ( 45 years) was chosen as cut-off value ( $<45$ years vs $\geq 45$ years).

\section{Allograft Handling and Surgical Technique}

All patients were operated using a nonanatomical doublebundle technique with nonirradiated Achilles tendon allograft. ${ }^{27}$ All the grafts were obtained from a single certified tissue bank (Banca delle cellule e del tessuto muscoloscheletrico, Bologna, Italy). The grafts were harvested in sterile fashion by four orthopedic surgeons and residents. At the end of the harvesting, before cadaver reconstruction, swabs for microbiological tests were obtained. Grafts were, therefore, stored at $-80^{\circ} \mathrm{C}$ until the date of the planned surgery, without undergoing gamma irradiation. In the operating room, the grafts were defrosted with hot water 30 minutes before the beginning of the surgical procedure, and further swabs were obtained. The grafts were then split in a "Y" shape, with the vertex separated from the calcaneal bone, which was eventually used for tunnel bone grafting. A 10$\mathrm{mm}$ tunnel was drilled through the tibia directed to the posteromedial part of the ACL footprint and a 7-mm tunnel was drilled from the anteromedial portal through the medial wall of the femoral condyle. After a lateral 3 to $5 \mathrm{~cm}$ incision to reach the posterior aspect of the capsule, both bundles of the graft were passed in the tibial tunnel, then one in the over-the-top position and one through the femoral tunnel. Finally, both were fixed to the femoral and tibial cortex with barbed metal staples.

All patients followed the same rehabilitation protocol, with return to unrestricted activity at 6 to 9 months.

\section{Statistical Analysis}

Statistical evaluation was performed using MedCalc (MedCalc software, Acacialaan 22, Ostend, Belgium). All the parametric continuous data were expressed as mean \pm standard deviation. The categorical data were expressed as absolute number and percentage. After the data have been tested for normal distribution, paired $t$-test was used to compare preoperative and postoperative values of Lysholm score. Chi-square was used to compare categorical variables.

Multiple regression analysis was performed using postoperative Lysholm score and as main outcomes. The regression analyses were performed in a stepwise fashion. Independent variables considered were age at primary reconstruction, age at revision, age at final follow-up, time from primary reconstruction to revision surgery, meniscus deficiency, final follow-up, more than one prior ACL reconstruction, combined procedures (except meniscectomy), preoperative Lysholm score, and graft donor age. Level of significance was set with $p<0.05$.

A posthoc power analysis was performed using means and standard deviations of the postoperative Lysholm score of the two groups based on allograft donor age $<45$ or $>45$ years. The $\alpha$ value was set at 0.05 and the power was calculated.

\section{Results}

Overall, 84 patients underwent revision ACL reconstruction in the considered time period. Eleven patients were excluded because a different graft was used, seven patients had previous contralateral ACL reconstruction, four patients 
12 Impact of Donor Age in ACL Reconstruction with Allografts Zaffagnini et al.

Table 1 Demographic and surgical details of the 52 patients included in the study

\begin{tabular}{|l|l|l|l|}
\hline Variables & Mean & SD & Range \\
\hline Age at primary ACL reconstruction (y) & 23.5 & 6.7 & $14-45$ \\
\hline Age at ACL revision (y) & 28.6 & 7.8 & $19-47$ \\
\hline Age at follow-up (y) & 33.3 & 7.7 & $23-50$ \\
\hline Time from primary ACL to revision (y) & 5.0 & 4.2 & $1-21$ \\
\hline Follow-up (y) & 4.8 & 0.8 & $4-7.6$ \\
\hline Allograft donor age (y) & 48.7 & 8.4 & $29-62$ \\
\hline Sex (male/female) & $48 / 4$ & $(92 \% / 8 \%)$ & \\
\hline Side (right/left) & $28 / 24$ & $(54 \% / 46 \%)$ & \\
\hline Meniscal status (intact/deficient) & $18 / 34$ & $(35 \% / 65 \%)$ & \\
\hline Combined procedure (yes/no) & $10 / 42$ & $(19 \% / 81 \%)$ & \\
\hline Multiple revision (yes/no) & $8 / 44$ & $(15 \% / 85 \%)$ & \\
\hline
\end{tabular}

Abbreviations: $\mathrm{ACL}$, anterior cruciate ligament, $\mathrm{SD}$, standard deviation, Note: Data are expressed as mean, $\mathrm{SD}$, and range $(\mathrm{ACL})$.

were $<18$ years old at time of revision, and two patients did not reach the minimum follow-up of 4 years. Of the 60 eligible patients, eight (13\%) patients were lost to followup. Therefore, the patients available at a mean $4.8 \pm 0.8$ years follow-up were 52 (-Table 1). Statistical analysis showed a significant improvement in the Lysholm score from $62.3 \pm 6.6$ at preoperative status to $84.4 \pm 12.3$ at final follow-up. The mean donor age was $48.7 \pm 8.4$ years; 14 patients $(27 \%)$ received a graft with an age $<45$ years, while 38 patients $(73 \%)$ received a graft with an age $\geq 45$ years. Baseline characteristics between the two groups were simi- lar; however, the Lysholm at final follow-up was significantly higher in patients who received a younger allograft $(p=0.0469)$ ( - Table 2).

The multiple regression model showed the graft's donor age ( - Fig. 1A), the follow-up length $(-$ Fig. 1B), and the preoperative Lysholm score ( - Fig. 1C) as a significant predictor of postoperative Lysholm score (-Table 3). Specifically, the value seemed to decrease approximately 5 points every 10 years of donor age increase. Regarding follow-up, the postoperative Lysholm value seemed to decrease approximately 5 points every 1 year after revision surgery.

Table 2 Comparison of demographic, surgical, and clinical details of the two subgroups of patients based on the allografts donor age $(<45$ y vs. $\geq 45$ y)

\begin{tabular}{|l|l|l|l|l|l|}
\hline \multirow{2}{*}{ Variables } & \multicolumn{2}{|l|}{$<\mathbf{4 5} \mathbf{y}(\boldsymbol{n}=\mathbf{1 4})$} & \multicolumn{2}{l}{$\mathbf{4 5} \mathrm{y}(\boldsymbol{n}=\mathbf{3 8})$} & \\
\cline { 2 - 5 } & Mean & SD & Mean & SD & \\
\hline Allograft donor age (y) & 38.1 & \pm 5.9 & 52.6 & \pm 5.2 & $<0.0001^{\text {a }}$ \\
\hline Age at primary ACL reconstruction (y) & 23.4 & \pm 7.3 & 23.6 & \pm 6.7 & $=0.9340$ \\
\hline Age at ACL revision (y) & 29.6 & \pm 9.1 & 28.2 & \pm 7.5 & $=0.6946$ \\
\hline Age at follow-up (y) & 33.8 & \pm 9.1 & 33.2 & \pm 7.4 & $=0.8457$ \\
\hline Time from primary ACL to revision (y) & 6.2 & \pm 7.1 & 4.6 & \pm 2.6 & $=0.1750$ \\
\hline Follow-up (y) & 4.4 & \pm 0.4 & 5.0 & \pm 1.1 & $=0.3941$ \\
\hline Sex (male/female) & $12 / 2$ & $36 / 2$ & & $=0.6199$ \\
\hline Side (right/left) & $8 / 6$ & $20 / 18$ & & $=0.7719$ \\
\hline Meniscal status (intact/deficient) & $3 / 11$ & $15 / 23$ & & $=0.2250$ \\
\hline Combined procedure (yes/no) & $1 / 13$ & $9 / 29$ & & $=0.1794$ \\
\hline Multiple revision (yes/no) & $3 / 11$ & $5 / 33$ & & $=0.4632$ \\
\hline Preoperative Lysholm score & 62.4 & \pm 4.3 & 62.3 & \pm 7.3 & $=0.6523$ \\
\hline Postoperative Lysholm score & 89.5 & \pm 3.2 & 80.1 & \pm 11.1 & $=0.0469^{\text {a }}$ \\
\hline
\end{tabular}

Abbreviations: $\mathrm{ACL}$, anterior cruciate ligament, SD, standard deviation,

Note: Data are expressed as mean, SD and $p$-Values (ACL).

${ }^{a}$ Statistically significant values $(p<0.05)$. 

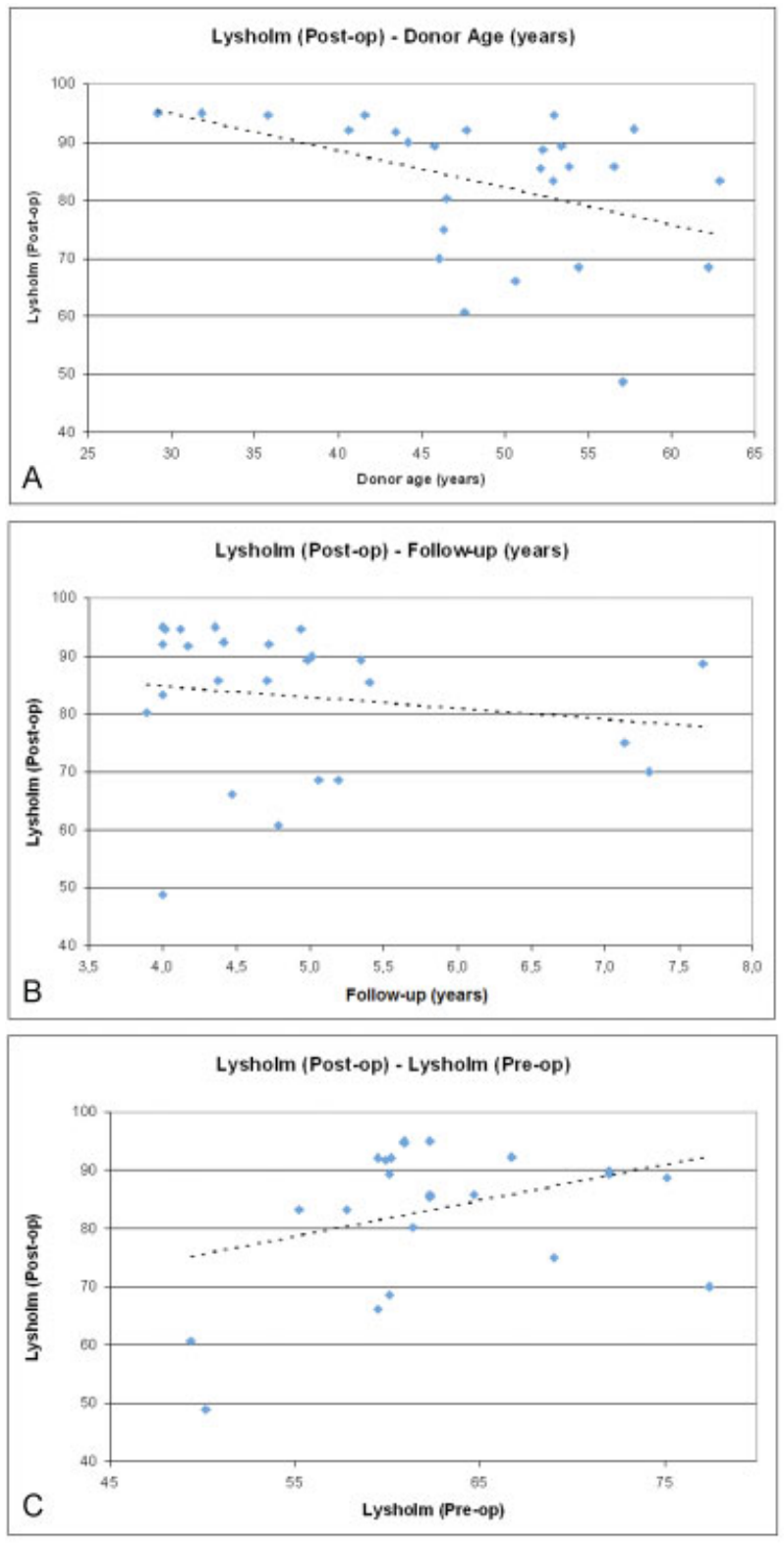

Fig. 1 Scatter plots graphics of the distribution of the postoperative Lysholm scores ( $y$-axis) based on donor age (A), follow-up (B), and preoperative Lysholm score $(C)$ at $x$-axis. The dotted line represents the tendency line.
In addition, the preoperative Lysholm score influenced almost 1.5 points of the postoperative value. The regression model was statistically significant $(p<0.05)$.

Posthoc power analysis for the postoperative Lysholm score showed a power of $99.7 \%$.

\section{Discussion}

The most important finding of the present study is that higher age of graft donor is associated with worse subjective clinical results at mid-term, after double-bundle ACL revision with nonirradiated Achilles tendon allograft. Furthermore, the length of the follow-up and the preoperative clinical status seemed to influence the postoperative results as well. However, the improvement in knee function did not substantially differ from what reported in other clinical studies. Pascual-Garrido et $\mathrm{al}^{28}$ reported an improvement of approximately 30 points of Lysholm scores using both nonirradiated patellar tendon and anterior tibialis at 2 to 4 years follow-up. The inferior improvement reported in the present study (22 points) could reflect the longer follow-up, as the multivariate analysis identified the follow-up as a variable that could influence negatively the clinical outcomes. Moreover, also the high number of meniscal lesions and combined procedure, summed to the presence of several multiple revisions, could have contributed.

The impact of donor age in ACL reconstruction with allografts still represents a controversial topic. Previous studies demonstrated the age-related decline in the biomechanical proprieties of the human ACL in the function of age. ${ }^{29,30}$ Conversely, the structural proprieties were reported to be independent from donor age for fresh-frozen bone-patellartendon-bone 20,21 and anterior and posterior tibialis allograft. $^{22,25}$ However, it should be remarked that only two studies $^{24,25}$ included donors older than 55 years, thus limiting the potential negative effects of tendon aging. Differently, Lewis and Shaw ${ }^{24}$ reported an age-related effect on the ultimate tensile strength, linear stiffness, and tangent modulus of human Achilles tendon allografts, thus recommending that when the allograft option is to be followed, Achilles tendon from young donors should be used whenever possible for the repair of the severely damaged tendon or ACL. This age-related Achilles tendon allograft behavior could be due to a different tissue quality according to donor age, as it has been demonstrated that Achilles tendon undergoes substantial aging-

Table 3 Multiple regression analysis: a coefficient $<0$ or $>0$ indicates respectively a negative or positive correlation of the variable with the main outcome

\begin{tabular}{|l|l|l|l|l|}
\hline Independent variables & Coefficient & SD & $r_{\text {partial }}$ & $p$-Value \\
\hline Allograft donor age & -0.546 & 0.2031 & -0.4972 & 0.0134 \\
\hline Follow-up & -4.7205 & 2.1264 & -0.4278 & 0.037 \\
\hline Preoperative Lysholm score & 1.5263 & 0.3545 & 0.6763 & 0.0003 \\
\hline
\end{tabular}

Abbreviations: $r_{\text {partial }}$, coefficient of correlation of the variable adjusted for the effect of the other variables in the model; SD, standard error. Note: Significance level: $p=0.0002$. 
induced changes that negatively affect the mechanical proprieties of in vivo tendon. ${ }^{31}$

Regarding clinical results, Hampton et $\mathrm{al}^{23}$ showed no influence of patellar-tendon allograft donor age, while the present study involving Achilles tendon allograft showed a decrease in clinical outcomes as donor age rises. This difference could be both due to the allograft tissue itself and to the fact that all the patients involved in the present study underwent revision $\mathrm{ACL}$, while the cohort of Hampton et $\mathrm{al}^{23}$ involved only primary isolated ACL reconstruction. It is reasonable that in patients with failed ACL reconstruction, the secondary stabilizer structures could have suffered higher damage than after primary ACL injury, thus placing more importance on the quality of the graft used to restore knee stability. Moreover, Hampton et $\mathrm{al}^{23}$ utilized both nonirradiated and low-dose irradiated allografts. The effects of irradiation (up to $28 \mathrm{kGy}$ ) have been in fact demonstrated to alter the structural and mechanical proprieties of the grafts, ${ }^{32}$ even if the use of low-dose irradiation seemed to mitigate such effect. ${ }^{22}$ However, Grassi et al $^{17}$ in their metaanalysis of 32 studies, regarding ACL revision, found out that outcomes between autografts and allografts were similar if studies regarding irradiated allografts (2 studies) were excluded.

Also, the type of graft and its biomechanical characteristics should not be ignored when comparing results of ACL reconstruction with allografts. It was in fact reported that the medial half of Achilles tendon allograft presents a lower failure load compared with anterior tibialis, posterior tibialis, peroneus longus, and hamstrings. ${ }^{33}$ It is reasonable that, however, often exceeding the strength of native $\mathrm{ACL}$, the strength of the allograft tissue decreases due to the avascular necrosis that occurs during healing and incorporation process, making sometimes the in vitro load to failure a misleading parameter to predict the in vivo behavior of the graft. In this regard, also the bone-tobone or soft-tissue-to-bone healing, and the graft manipulation or configuration (thick single-bundle vs. splitted double bundle) could play a non-negligible role. Therefore, unless well-designed controlled studies comparing Achilles tendon and other graft types, no conclusions can be drawn regarding the effect of donor age on clinical results according to graft type.

Lastly, it should be mentioned that also the preoperative patient status and knee function could influence the outcome; specifically, patients with less disability were more likely to present final higher functional scores after ACL revision. It should be acknowledged that the nine-point difference found between patients that received younger or older allograft could represent a borderline value for clinical significance, as the minimum clinically important difference for Lysholm score has not been determined. ${ }^{34}$

The present study presents several limitations: the limited number of patients could have failed to detect significant findings regarding the less represented variables, like combined procedures and multiple revisions. In addition, the Lysholm score has not yet been validated in Italian language and being a patient-reported clinical score, language com- prehension could represent a bias in score evaluation. However, the main strength of the present study is represented by the fact that all ACL revision were performed with the same technique by the two senior surgeons using allograft tissue provided by a single certified tissue bank, thus receiving the same handling process.

In conclusion, the age of allograft donor, length of followup, and preoperative clinical status seem to be predictors of mid-term results after revision ACL with nonirradiated Achilles tendon allograft. Especially, as older graft donor was a negative predictor for final functional outcomes, when using Achilles tendon allografts, young donors should be used for the present double-bundle revision ACL reconstruction.

\section{References}

1 Eagan MJ, McAllister DR. Biology of allograft incorporation. Clin Sports Med 2009;28(02):203-214

2 Wilde J, Bedi A, Altchek DW. Graft selection for revision ACL reconstruction. In: Marx RG, ed. Revision ACL Reconstruction. New York: Springer; 2014:75-86

3 Wright RW, Huston LJ, Spindler KP, et al; MARS Group. Descriptive epidemiology of the Multicenter ACL Revision Study (MARS) cohort. Am J Sports Med 2010;38(10):1979-1986

4 Buda R, Ruffilli A, Di Caprio F, et al. Allograft salvage procedure in multiple-revision anterior cruciate ligament reconstruction. Am J Sports Med 2013;41(02):402-410

5 Chougule S, Tselentakis G, Stefan S, Stefanakis G. Revision of failed anterior cruciate ligament reconstruction with quadrupled semitendinosus allograft: intermediate-term outcome. Eur J Orthop Surg Traumatol 2015;25(03):515-523

6 Fox JA, Pierce M, Bojchuk J, Hayden J, Bush-Joseph CA, Bach BR Jr. Revision anterior cruciate ligament reconstruction with nonirradiated fresh-frozen patellar tendon allograft. Arthroscopy 2004; 20(08):787-794

7 Grassi A, Zaffagnini S, Marcheggiani Muccioli GM, Neri MP, Della Villa S, Marcacci M. After revision anterior cruciate ligament reconstruction, who returns to sport? A systematic review and meta-analysis. Br J Sports Med 2015;49(20):1295-1304

8 Zeng C, Gao SG, Li H, et al. Autograft versus allograft in anterior cruciate ligament reconstruction: a meta-analysis of randomized controlled trials and systematic review of overlapping systematic reviews. Arthroscopy 2016;32(01):153-163

9 MARS Group; MARS Group. Effect of graft choice on the outcome of revision anterior cruciate ligament reconstruction in the Multicenter ACL Revision Study (MARS) cohort. Am J Sports Med 2014; 42(10):2301-2310

10 Jackson DW, Simon TM, Kurzweil PR, Rosen MA. Survival of cells after intra-articular transplantation of fresh allografts of the patellar and anterior cruciate ligaments. DNA-probe analysis in a goat model. J Bone Joint Surg Am 1992;74(01):112-118

11 Jackson DW, Grood ES, Goldstein JD, et al. A comparison of patellar tendon autograft and allograft used for anterior cruciate ligament reconstruction in the goat model. Am J Sports Med 1993;21(02): 176-185

12 Malinin TI, Levitt RL, Bashore C, Temple HT, Mnaymneh W. A study of retrieved allografts used to replace anterior cruciate ligaments. Arthroscopy 2002;18(02):163-170

13 McAllister DR, Joyce MJ, Mann BJ, Vangsness CT Jr. Allograft update: the current status of tissue regulation, procurement, processing, and sterilization. Am J Sports Med 2007;35(12): 2148-2158

14 Vangsness CT Jr, Dellamaggiora RD. Current safety sterilization and tissue banking issues for soft tissue allografts. Clin Sports Med 2009;28(02):183-189 
15 Fideler BM, Vangsness CT Jr, Lu B, Orlando C, Moore T. Gamma irradiation: effects on biomechanical properties of human bonepatellar tendon-bone allografts. Am J Sports Med 1995;23(05): 643-646

16 Lamblin CJ, Waterman BR, Lubowitz JH. Anterior cruciate ligament reconstruction with autografts compared with non-irradiated, non-chemically treated allografts. Arthroscopy 2013;29 (06):1113-1122

17 Grassi A, Kim C, Marcheggiani Muccioli GM, Zaffagnini S, Amendola A. What is the mid-term failure rate of revision ACL reconstruction? A systematic review. Clin Orthop Relat Res 2017;475 (10):2484-2499

18 Erickson BJ, Cvetanovich GL, Frank RM, Riff AJ, Bach BR Jr. Revision ACL reconstruction: a critical analysis review. JBJS Rev 2017;5 (06): 1

19 Allen CR, Anderson AF, Cooper DE, et al; MARS Group. Surgical predictors of clinical outcomes after revision anterior cruciate ligament reconstruction. Am J Sports Med 2017;45(11):2586-2594

20 Blevins FT, Hecker AT, Bigler GT, Boland AL, Hayes WC. The effects of donor age and strain rate on the biomechanical properties of bone-patellar tendon-bone allografts. Am J Sports Med 1994;22 (03):328-333

21 Flahiff CM, Brooks AT, Hollis JM, Vander Schilden JL, Nicholas RW. Biomechanical analysis of patellar tendon allografts as a function of donor age. Am J Sports Med 1995;23(03):354-358

22 Greaves LL, Hecker AT, Brown CH Jr. The effect of donor age and low-dose gamma irradiation on the initial biomechanical properties of human tibialis tendon allografts. Am J Sports Med 2008;36 (07):1358-1366

23 Hampton DM, Lamb J, Klimkiewicz JJ. Effect of donor age on patellar tendon allograft ACL reconstruction. Orthopedics 2012; 35(08):e1173-e1176

24 Lewis G, Shaw KM. Tensile properties of human tendo Achillis: effect of donor age and strain rate. J Foot Ankle Surg 1997;36(06): 435-445

25 Swank KR, Behn AW, Dragoo JL. The effect of donor age on structural and mechanical properties of allograft tendons. Am J Sports Med 2015;43(02):453-459
26 Lysholm J, Gillquist J. Evaluation of knee ligament surgery results with special emphasis on use of a scoring scale. Am J Sports Med 1982;10(03):150-154

27 Marcacci M, Zaffagnini S, Bonanzinga T, Marcheggiani Muccioli GM, Bruni D, Iacono F. Over-the-top double-bundle revision ACL reconstruction. Knee Surg Sports Traumatol Arthrosc 2012;20 (07):1404-1408

28 Pascual-Garrido C, Carbo L, Makino A. Revision of anterior cruciate ligament reconstruction with allografts in patients younger than 40 years old: a 2 to 4 year results. Knee Surg Sports Traumatol Arthrosc 2014;22(05):1106-1111

29 Noyes FR, Grood ES. The strength of the anterior cruciate ligament in humans and Rhesus monkeys. J Bone Joint Surg Am 1976;58 (08):1074-1082

30 Woo SL, Hollis JM, Adams DJ, Lyon RM, Takai S. Tensile properties of the human femur-anterior cruciate ligament-tibia complex. The effects of specimen age and orientation. Am J Sports Med 1991;19(03):217-225

31 Onambele GL, Narici MV, Maganaris CN. Calf muscle-tendon properties and postural balance in old age. J Appl Physiol (1985) 2006; 100(06):2048-2056

32 Balsly CR, Cotter AT, Williams LA, Gaskins BD, Moore MA, Wolfinbarger L Jr. Effect of low dose and moderate dose gamma irradiation on the mechanical properties of bone and soft tissue allografts. Cell Tissue Bank 2008;9(04):289-298

33 Arnout N, Myncke J, Vanlauwe J, Labey L, Lismont D, Bellemans J. The influence of freezing on the tensile strength of tendon grafts: a biomechanical study. Acta Orthop Belg 2013;79(04):435-443

34 Collins NJ, Misra D, Felson DT, Crossley KM, Roos EM. Measures of knee function: International Knee Documentation Committee (IKDC) Subjective Knee Evaluation Form, Knee Injury and Osteoarthritis Outcome Score (KOOS), Knee Injury and Osteoarthritis Outcome Score Physical Function Short Form (KOOS-PS), Knee Outcome Survey Activities of Daily Living Scale (KOS-ADL), Lysholm Knee Scoring Scale, Oxford Knee Score (OKS), Western Ontario and McMaster Universities Osteoarthritis Index (WOMAC), Activity Rating Scale (ARS), and Tegner Activity Score (TAS). Arthritis Care Res (Hoboken) 2011;63(Suppl 11):S208-S228 